\title{
May 2018 Imaging Case of the Month
}

\author{
Michael B. Gotway, MD \\ Department of Radiology \\ Mayo Clinic Arizona \\ Scottsdale, AZ USA
}

Clinical History: A 79-year-old man with a past medical history significant for mild, intermittent asthma since childhood and mild aortic stenosis presents to the Emergency Room with fevers and chills for 5 days, associated with dry cough and dyspnea on exertion. His past medical history was otherwise relatively unremarkable, with coronary artery disease as evidenced by coronary artery calcium at a calcium scoring CT, hypothyroidism, and dyslipidemia. The patient has allergies to dust and penicillin, and his only medications included thyroid replacement, aspirin, and an albuterol inhaler as needed. He was a 15-pack-year smoker, quitting 30 years ago. His past surgical history was remarkable only for tonsillectomy, inguinal hernia repair, meniscal repair, and sigmoid colon resection for diverticular abscess 14 years earlier. The patient was afebrile, his heart rate was 96 beats / minute and regular, decreased breath sounds at the lung bases was noted, and the white blood cell count was normal.

Electrocardiography showed no abnormalities. Oxygen saturation was $92 \%$ on room air. Frontal chest radiography (Figures $1 \mathrm{~A}$ and $\mathrm{B}$ ) was performed.

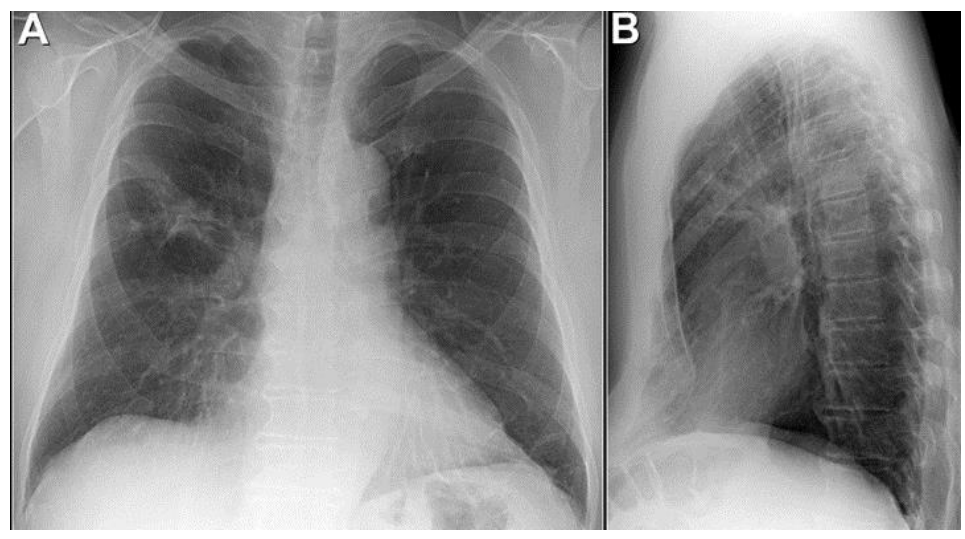

Figure 1. Frontal $(A)$ and lateral $(B)$ chest radiography.

Which of the following represents the most accurate assessment of the frontal chest imaging findings?

1. Chest frontal imaging shows bilateral pleural fluid collections

2. Chest radiography shows bilateral lower lobe bronchial wall thickening and patchy consolidation

3. Chest radiography shows cavitary lung disease

4. Chest radiography shows numerous small nodules

5. Chest radiography shows peribronchial and mediastinal lymphadenopathy 


\section{Correct! \\ 2. Chest radiography shows bilateral lower lobe bronchial wall thickening and patchy consolidation}

Chest radiography shows patchy, bilateral bibasilar consolidation and bronchovascular thickening, but without evidence of peribronchial or mediastinal lymph node enlargement, other nodules, or pleural disease. No miliary nodules are evident.

The patient underwent CT of the abdomen and pelvis (Figure 2), evidently searching for a source for his complaints in the context of some abdominal tenderness as well.

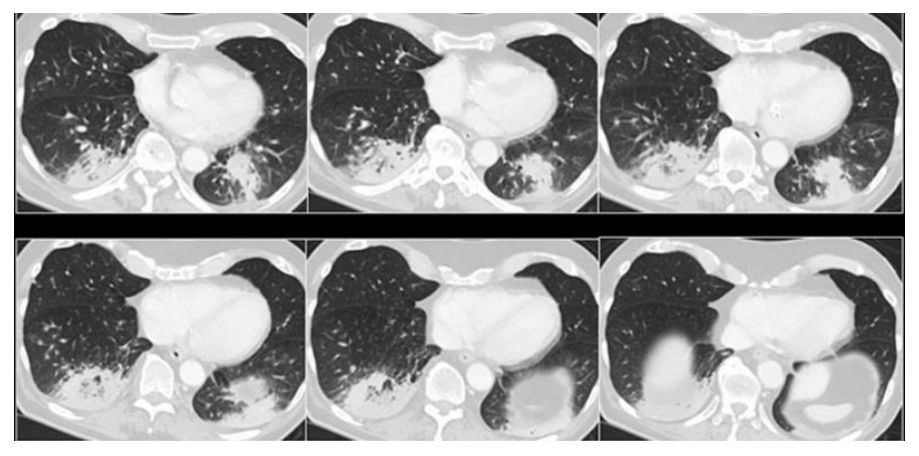

Figure 2. Representative images from lung base images from CT of the abdomen shows bilateral lower lobe consolidation consistent with bronchopneumonia. This study was negative for any intra-abdominal process.

This study showed findings consistent with bibasilar bronchopneumonia. The patient was administered intravenous levofloxacin and admitted to the hospital, and then switched to ceftriaxone and azithromycin. The patient improved and his was discharged a few days later on oral antibiotics. Thoracic CT (Figure 3) was performed 12 days later.

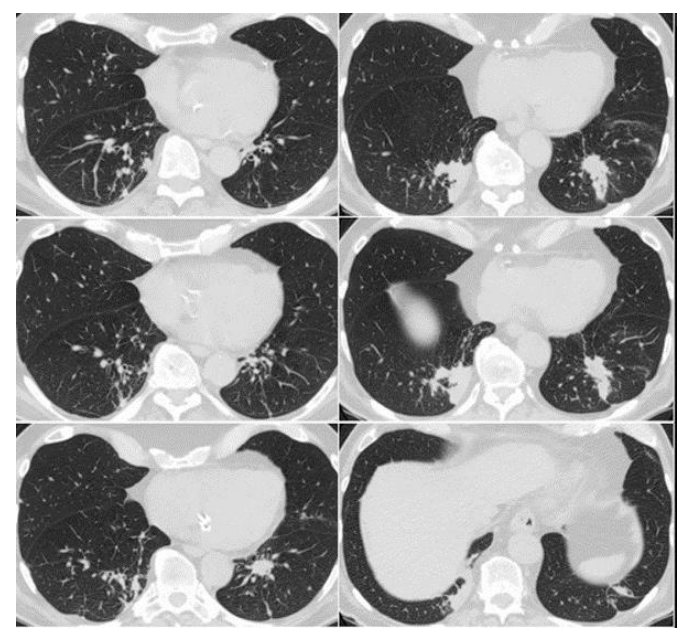

Figure 3. Representative images from the axial unenhanced thoracic CT performed 12 days following the abdominal CT (Figure 2) shows bilateral lower lobe nodularappearing consolidation which is improved from the abdominal CT. 
Which of the following represents the most accurate assessment of the thoracic CT findings?

1. Thoracic CT shows development of cavitary pulmonary lesions

2. Thoracic CT shows improvement in the bibasilar opacities

3. Thoracic CT shows new multifocal ground glass opacity

4. Thoracic CT shows no change in the bibasilar opacities

5. Thoracic CT shows worsening of the bibasilar opacities 


\section{Correct! \\ 2. Thoracic CT shows improvement in the bibasilar opacities}

Thoracic CT shows that the bibasilar opacities seen at CT abdomen 12 days earlier are still present, somewhat nodular in character, but have improved considerably.

The patient underwent testing for coccidioidomycosis, which was negative. Repeat thoracic CT (Figure 4) was performed about 1 month later.

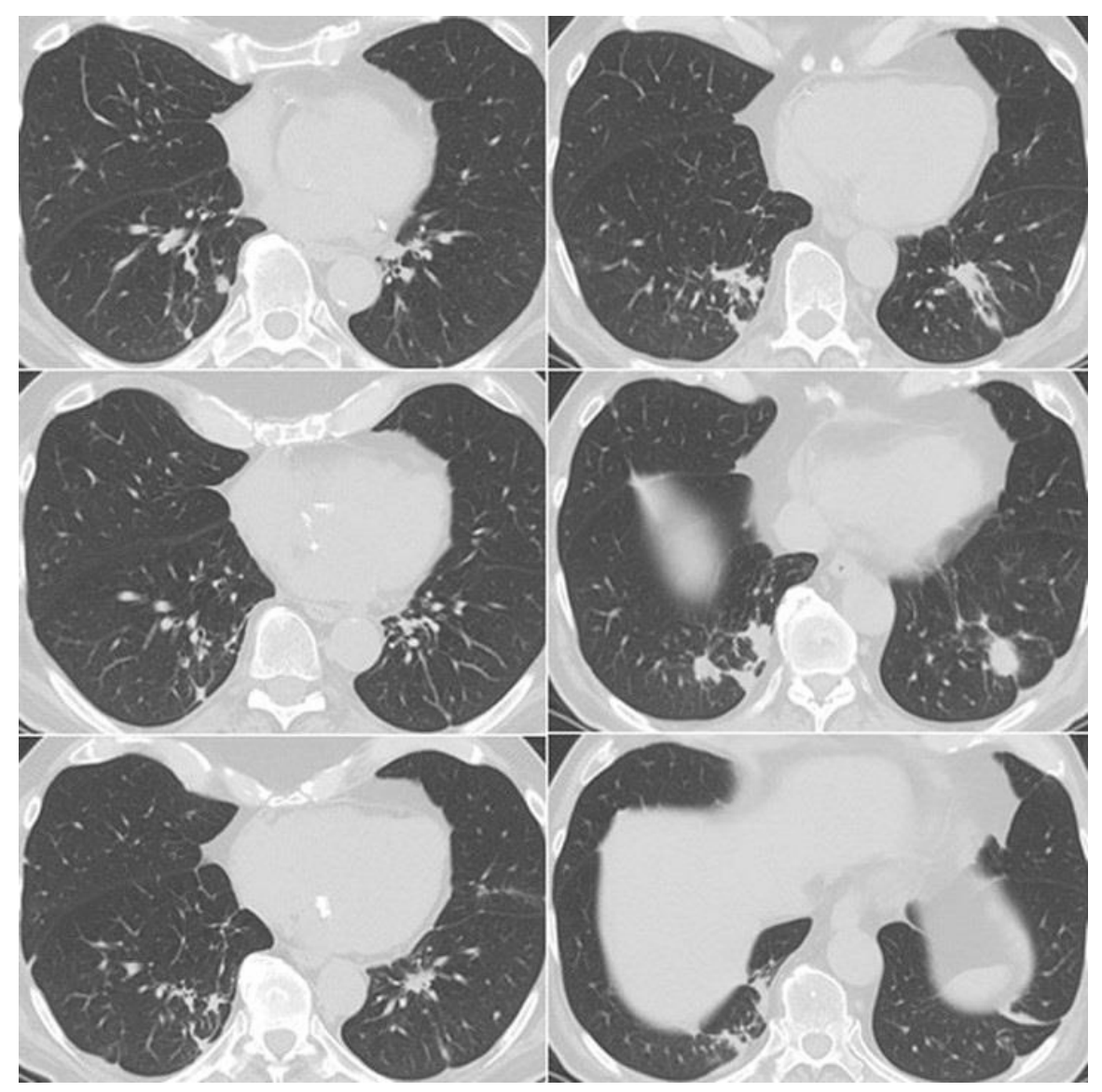

Figure 4. Representative images from the axial unenhanced thoracic CT performed over 1.5 months following the abdominal CT (Figure 2) and about 1 month following Figure 3 shows continued improvement in the bilateral lower lobe nodular-appearing consolidation.

Which of the following represents the most accurate assessment of the thoracic CT findings?

1. Thoracic CT shows continued interval improvement in the bibasilar opacities

2. Thoracic CT shows development of cavitary pulmonary lesions

3. Thoracic CT shows migration of the bibasilar opacities

4. Thoracic CT shows new numerous nodules

5. Thoracic CT shows worsening of the bibasilar opacities 


\section{Correct! \\ 1. Thoracic CT shows continued interval improvement in the bibasilar opacities}

Thoracic CT shows that the bibasilar opacities seen at CT abdomen at presentation (Figure 2) and on follow up CT (Figure 3) continue to improve, with no new or migratory pulmonary opacities and no evidence of new or increasing lymph node enlargement.

The patient underwent yet another thoracic CT, now about 5 months following initial presentation (Figure 5), which showed near-complete resolution of the bibasilar pneumonia.

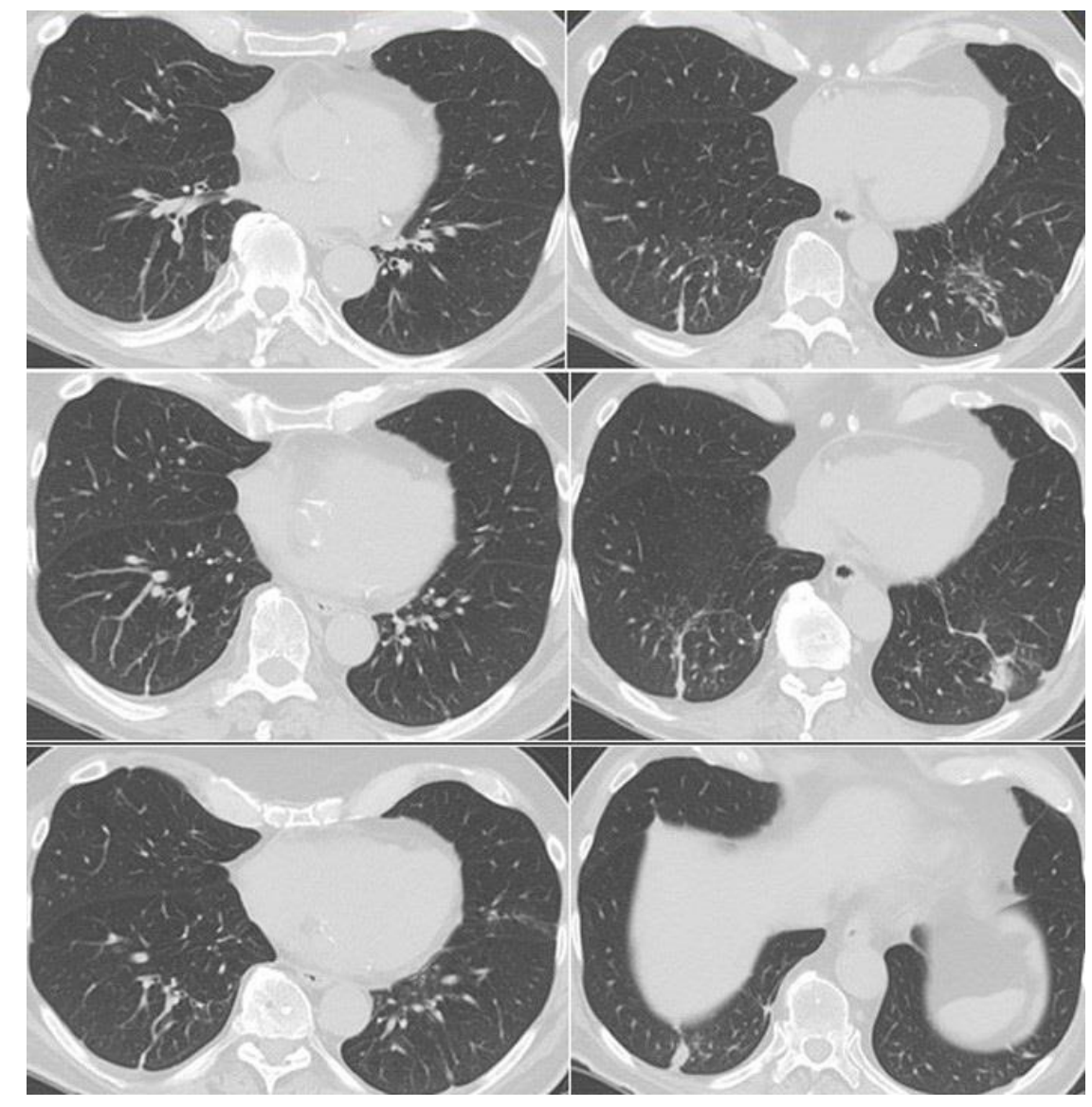

Figure 5. Representative images from the axial unenhanced thoracic CT performed over 5 months following the initial presentation abdominal CT (Figure 2) and nearly 4 months following Figure 3 shows near complete resolution in the bilateral lower lobe nodularappearing consolidation.

The patient clinically improved as well, and did well for the next 8 months, at which time he again presented with cough, shortness of breath, and some malaise, unimproved with another course of azithromycin, to which corticosteroids were added. During this time the patient presented to the Emergency Room with the same complaints, and his oxygen saturation was noted to be $93 \%$ on room air, decreasing to $88 \%$ with hallway exercise. Frontal and lateral chest radiography (Figure 6) was performed. 


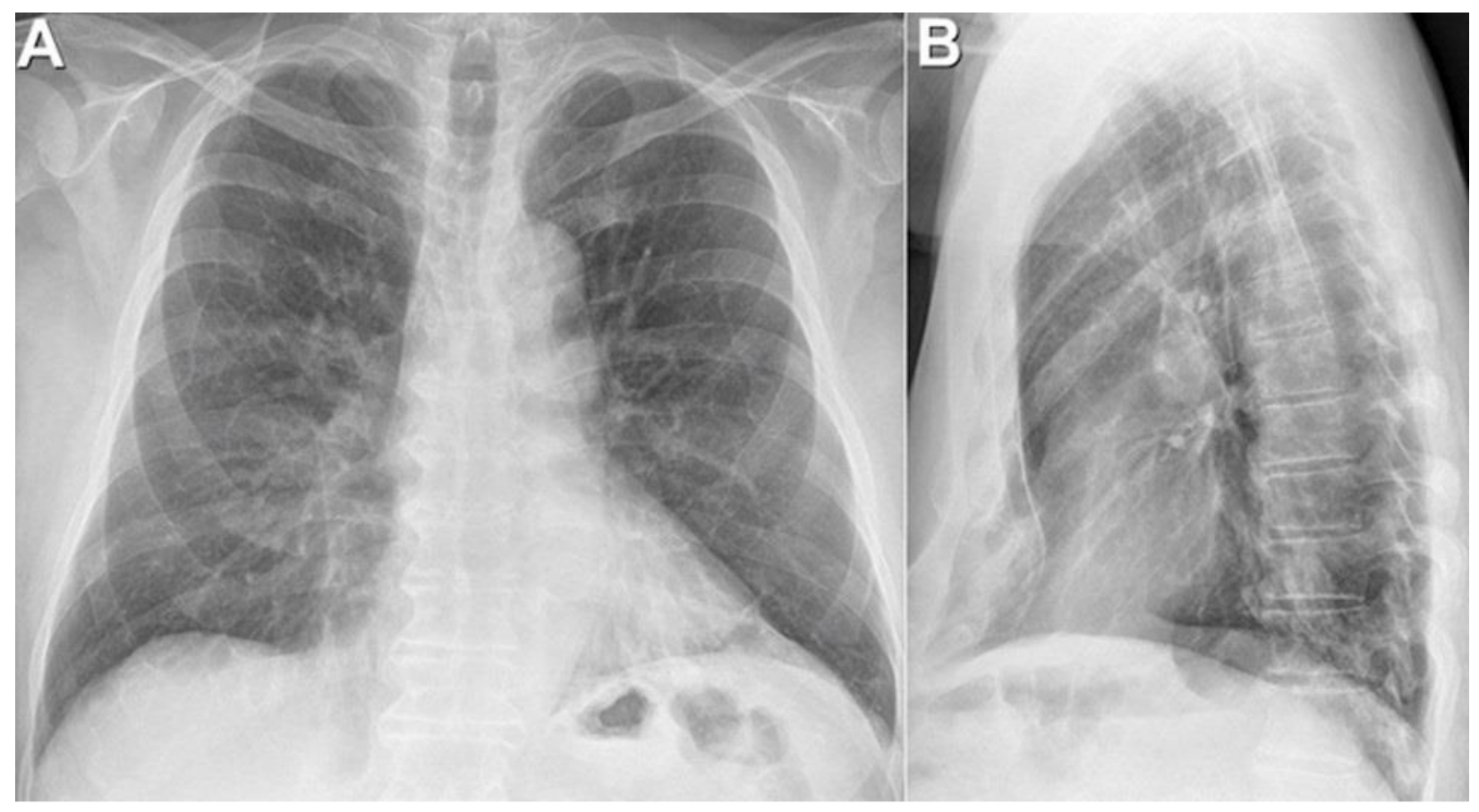

Figure 6. Chest radiography after recurrence of symptoms.

Which of the following represents the most accurate assessment of the chest radiographic findings?

1. Frontal and lateral chest radiography shows left lower lobe consolidation

2. Frontal and lateral chest radiography shows loculated pleural fluid

3. Frontal and lateral chest radiography shows new mediastinal widening

4. Frontal and lateral chest radiography shows new small nodules

5. Frontal and lateral chest radiography shows pneumothorax 


\section{Correct!}

\section{Frontal and lateral chest radiography shows left lower lobe consolidation}

Frontal and lateral chest radiography shows patchy left lower lobe bronchovascular thickening and consolidation suggesting lower lobe pneumonia. The cardiomediastinal contours are not abnormal and appear stable, and no pleural abnormalities (pleural effusion or pneumothorax) are seen. No new nodules are present.

The patient underwent thoracic CTA performed for pulmonary embolism evaluation (Figure 7) while in the Emergency Room.

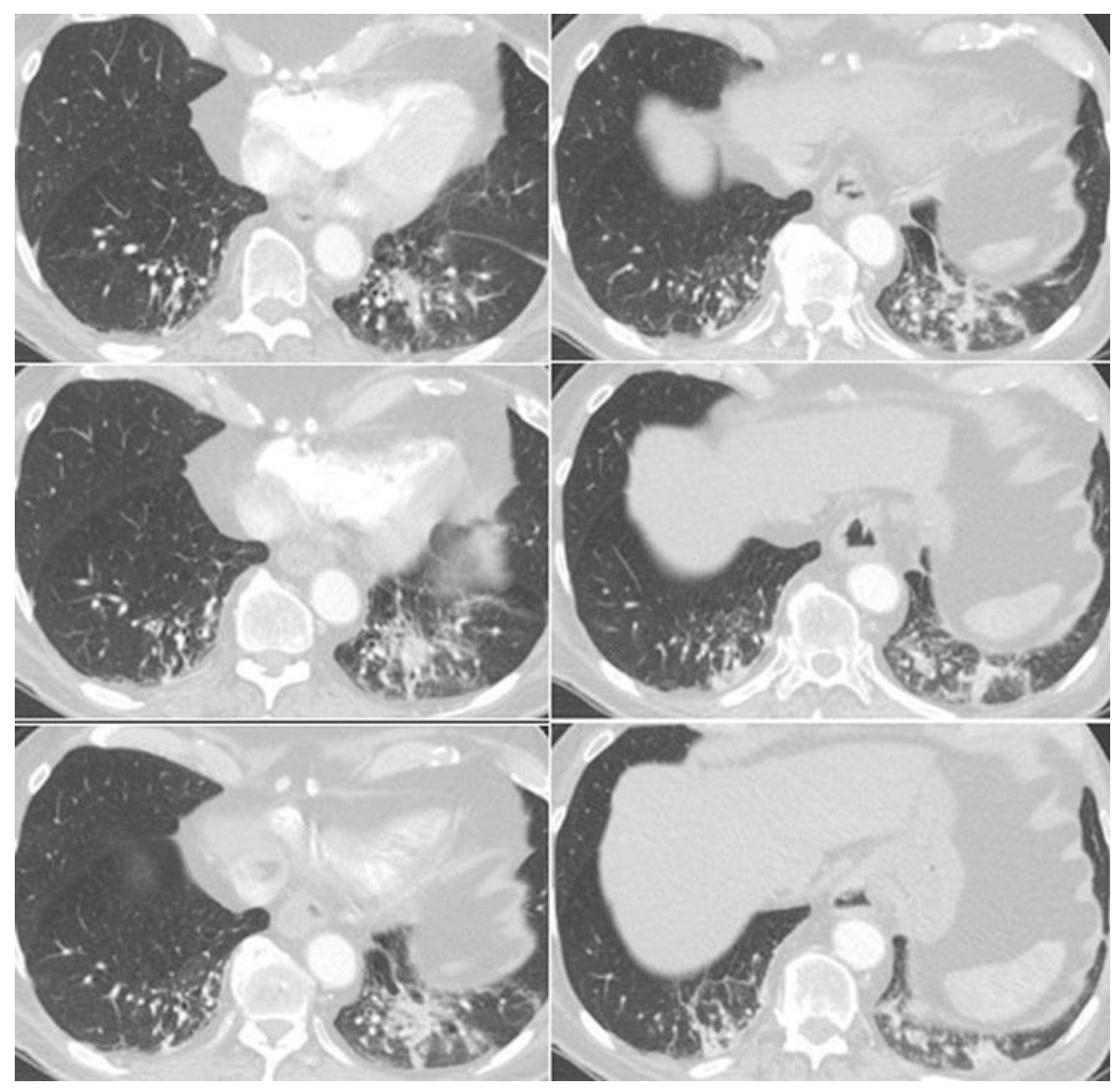

Figure 7. Representative images from the axial thoracic CTA performed for pulmonary embolism evaluation.

Which of the following represents the most accurate assessment of the thoracic CTA findings?

1. Thoracic CTA shows bilateral pulmonary emboli with right ventricular strain

2. Thoracic CTA shows development of cavitary pulmonary lesions

3. Thoracic CTA shows new numerous disseminated nodules

4. Thoracic CTA shows new upper lobe consolidation

5. Thoracic CTA shows recurrence of bilateral lower lobe pneumonia 


\section{Correct! \\ 5. Thoracic CTA shows recurrence of bilateral lower lobe pneumonia}

Thoracic CTA shows no evidence of pulmonary embolism. No new upper lobe consolidation or disseminated nodules are seen, but left-greater-than-right bilateral lower lobe bronchovascular thickening and patchy consolidation is now present.).

The patient was treated again with levofloxacin and seen by community internal medicine 8 days later. The patient claimed no improvement in his symptoms, although his physician thought he had improved and there were no signs of infection currently. There was a thought that the patient "was not doing well" on his asthma therapy, and his inhalers (fluticasone and ipratropium bromide) were increased. Repeat frontal and lateral chest radiography (Figure 8) was performed.

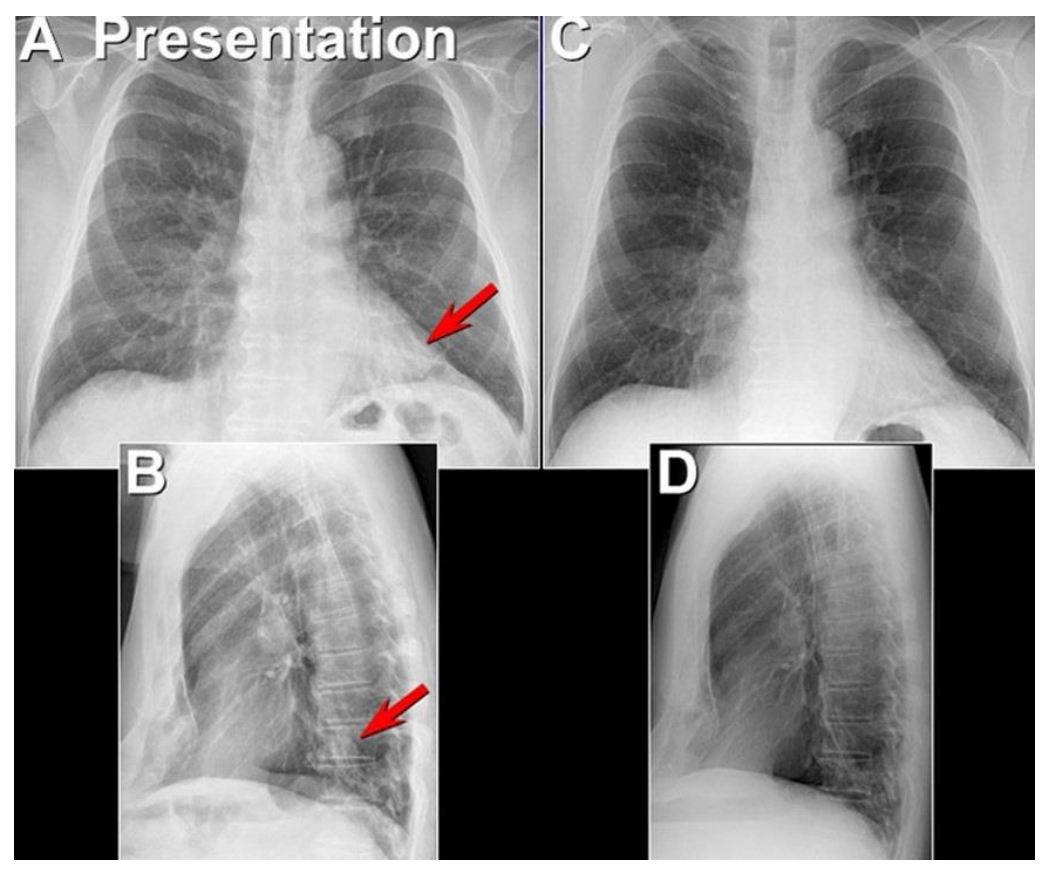

Figure 8. Frontal and lateral chest radiography at Emergency Room presentation ( $A$ and $B)$ and 8 days later following levofloxacin therapy (C and $D)$.

Which of the following represents the most accurate assessment of the chest radiographic findings?

1. Frontal and lateral chest radiography shows clearing of the bibasilar opacities

2. Frontal and lateral chest radiography shows developing pleural disease

3. Frontal and lateral chest radiography shows new peribronchial and mediastinal lymph node enlargement

4. Frontal and lateral chest radiography shows no interval change from the Emergency Room presentation chest radiograph (Figure 6)

5. Frontal and lateral chest radiography shows worsening multifocal consolidation bilaterally 


\section{Correct!}

\section{Frontal and lateral chest radiography shows clearing of the bibasilar opacities}

Repeat frontal and lateral chest radiography shows the left-greater-than-right, bilateral, lower lobe bronchovascular thickening and consolidation suggesting lower lobe pneumonia on the Emergency Room presentation chest radiograph (Figure 6) has largely resolved. No pleural abnormality or evidence of new pulmonary opacities or lymph node enlargement is present.

The patient was seen about 1 month later by pulmonary medicine.

Which of the following diagnoses is the most appropriate consideration for this patient at this point?

1. Aspiration pneumonia

2. Amyloidosis

3. Bronchogenic malignancy

4. Langerhans cell histiocytosis

5. Lymphoproliferative disorder 


\section{Correct! \\ 1. Aspiration pneumonia}

The presence of recurrent bilateral lower lobe pneumonias in an older patient raises concern for aspiration pneumonia. Neither lymphoproliferative disorder nor bronchogenic malignancy would be expected to regress following antibiotic therapy directed towards community-acquired pneumonia. Similarly, while amyloidosis could produce nodular pulmonary opacities, this patient has no history of renal disease and pulmonary opacities due to amyloidosis would not wax and wane in this fashion, nor would they be expected to respond to antibiotic therapy. Langerhans cell histiocytosis typically produces upper lobe nodular and cystic disease, typically nearly completely cystic in a patient of this age, and the patient has not been an active smoker for 30 years.

The patient underwent maxillofacial CT to assess for sinus disease, which was negative, and pulmonary medicine felt there was nothing else to do as regards possible recurrent aspiration pneumonia at this point. About one month following pulmonary medicine consultation, the patient again presented to the Emergency Room with the same symptoms as before and underwent repeat chest radiography (not shown), which showed possible right lower lobe pneumonia. He was treated with clarithromycin and was not seen for about 10 more months. He then re-presented to his primary care physician with complaints of asthma-like symptoms, for which his inhaled steroids were re-started, his ipratropium bromide inhaler dose was increased, and he was again encouraged to use his adrenergic bronchodilator as needed. A repeat chest radiograph was performed (image not shown) and was interpreted as negative for acute disease. Over the ensuing year the patient was seen by various consultants, such as an ophthalmologist for cataracts, an allergist for his atopic symptoms, and a dermatologist for resection of a localized squamous cell carcinoma on one of his forearms. After nearly a year, the patient re-presented to the Emergency Room for complaints of generalized weakness, progressive exertional dyspnea, and cough productive of white sputum, for which repeat thoracic CTA (Figure 9) was performed.

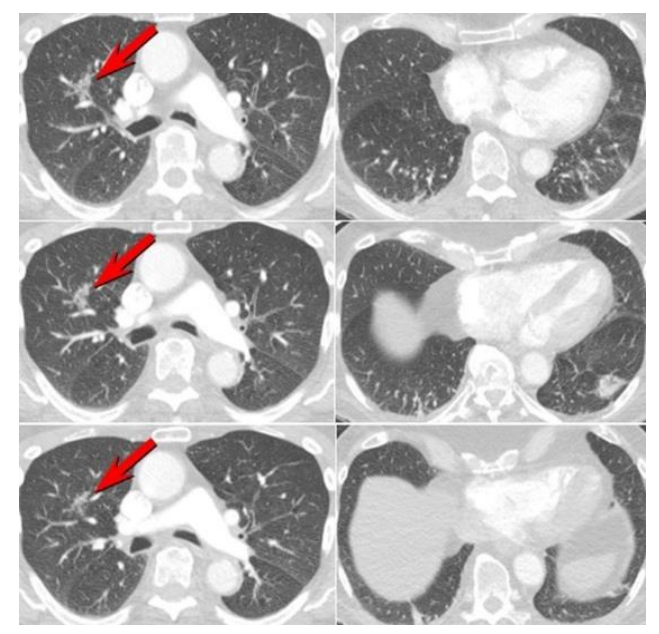

Figure 9. Thoracic CTA performed for pulmonary embolism. 
Which of the following represents the most accurate assessment of the thoracic CTA findings?

1. Thoracic CTA shows bilateral pulmonary emboli with right ventricular strain

2. Thoracic CTA shows development of scattered new small pulmonary nodules

3. Thoracic CTA shows new multifocal consolidation

4. Thoracic CTA shows new upper lobe localized nodular opacities

5. Thoracic CTA shows recurrence of the bilateral lower lobe pneumonia 


\section{Correct! \\ 4. Thoracic CTA shows new upper lobe localized nodular opacities}

Thoracic CTA shows no evidence of pulmonary embolism. No recurrence of the bilateral lower lobe pneumonia or nodules is seen, but a small cluster of new nodular opacities is now present within the central anterior segment of the right upper lobe. No other areas of consolidation or new small scattered nodules are evident.

One month later the patient experienced complaints of left eye blurry vision, dizziness, non-focal weakness, which occurred again 8 days later, both episodes resolving within an hour. Brain CT and CTA (not shown) was negative. The patient was then seen again in 8 months when he presented for a routine annual exam, for which no new issues were noted. He then disappeared from follow up for a further 9 months, until he represented to his primary care physician for complaints of shortness of breath and constipation for 2-3 weeks' duration. An ECG and laboratory data, including a complete blood count, BMP, d-dimer, electrolyte panel, TSH, and renal function, were within normal limits. A repeat frontal and lateral chest radiograph (Figure 10) was performed.

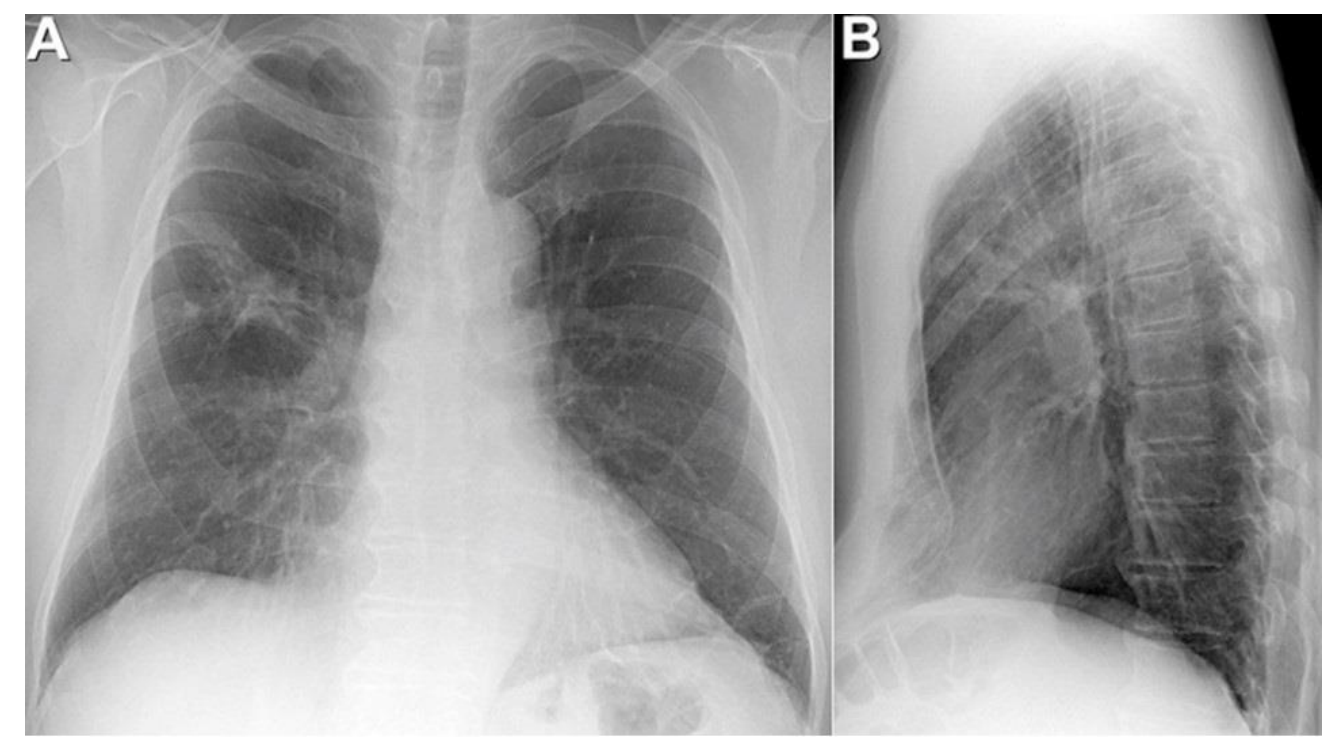

Figure 10. Frontal and lateral chest radiography.

Which of the following represents the most accurate assessment of the chest radiographic findings?

1. Frontal and lateral chest radiography shows developing pleural disease

2. Frontal and lateral chest radiography shows new cavitary lung disease

3. Frontal and lateral chest radiography shows new focal lung opacity

4. Frontal and lateral chest radiography shows no interval change from the most recent chest radiograph (Figure $8 \mathrm{C}$ and $\mathrm{D}$ ) is seen

5. Frontal and lateral chest radiography shows recurrent bilateral lower lobe pneumonia 


\section{Correct!}

\section{Frontal and lateral chest radiography shows new focal lung opacity}

Frontal and lateral chest radiography (Figure 10) shows new focal lung opacity within the right upper lobe. No recurrence of the previous bilateral lower lobe pneumonias or nodules is seen, nor is there evidence of cavitation or pleural disease. No other areas of consolidation or new small scattered nodules are evident.

Thoracic CTA using a pulmonary embolism protocol (Figure 11) was again performed.

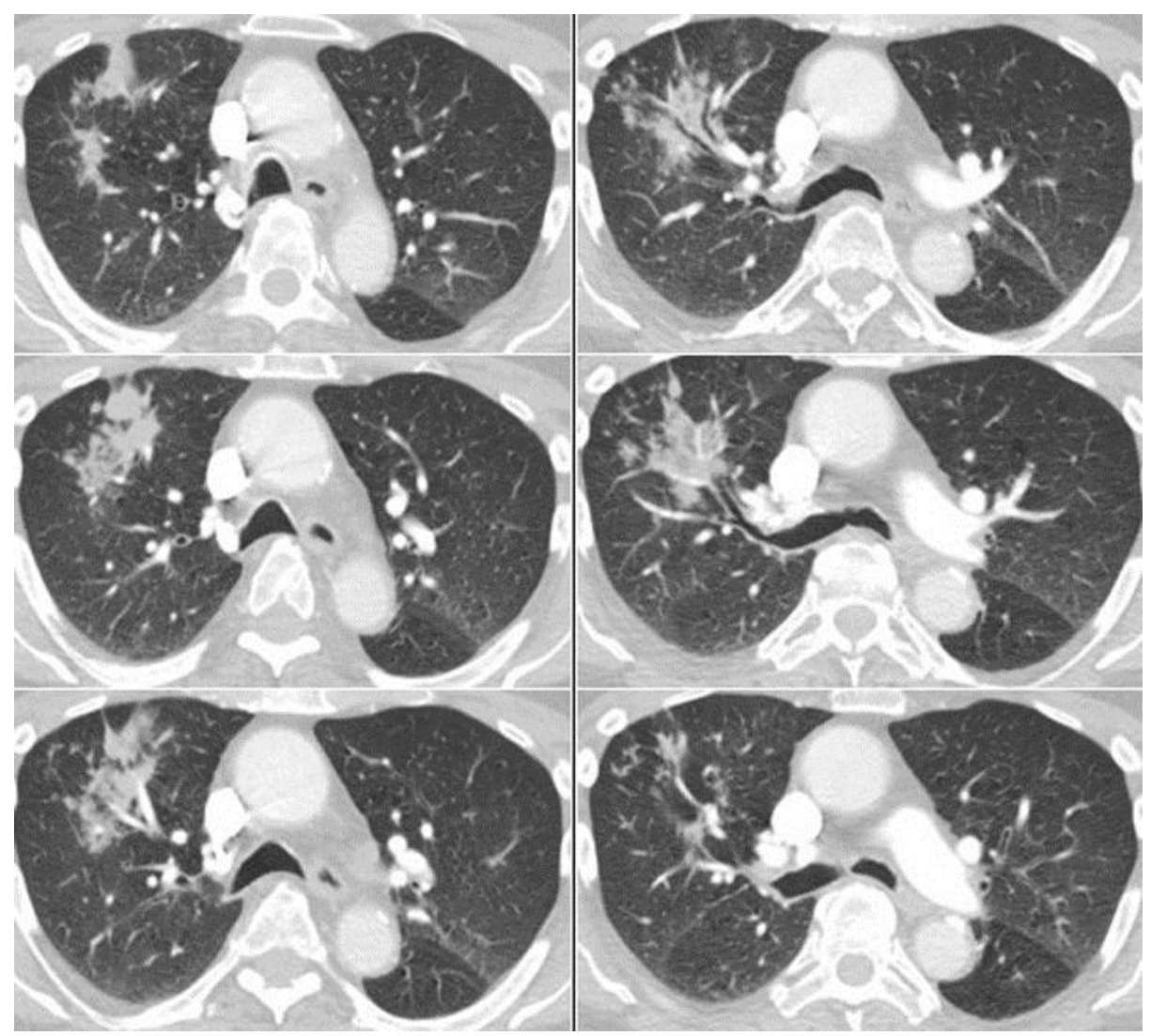

Figure 11. Representative images from the thoracic CTA performed for pulmonary embolism evaluation.

Which of the following represents the most accurate assessment of the thoracic CTA findings?

1. Thoracic CTA shows bilateral pulmonary emboli with right ventricular strain

2. Thoracic CTA shows development of scattered new small pulmonary miliary nodules

3. Thoracic CTA shows new pleural thickening and loculated pleural liquid

4. Thoracic CTA shows progression of the anterior segment right upper lobe localized nodular opacities seen on the prior thoracic CTA 17 months previously

5. Thoracic CTA shows recurrence of the bilateral lower lobe pneumonia 


\section{Correct! \\ 4. Thoracic CTA shows progression of the anterior segment right upper lobe localized nodular opacities seen on the prior thoracic CTA 17 months previously}

Thoracic CTA shows no evidence of pulmonary embolism. No recurrence of the bilateral lower lobe pneumonia or nodules is seen, but the nodular opacities within the central anterior segment of the right upper lobe on the study about 17 months earlier (Figure 9) have progressed substantially. No miliary nodules are seen and no pleural disease is present. No other areas of consolidation are evident.

The patient was presumed to have community-acquired bronchopneumonia and was started on levofloxacin. Chest radiography (Figure 12) was repeated after about 1.5 months, after which the patient said he felt "back to his baseline."

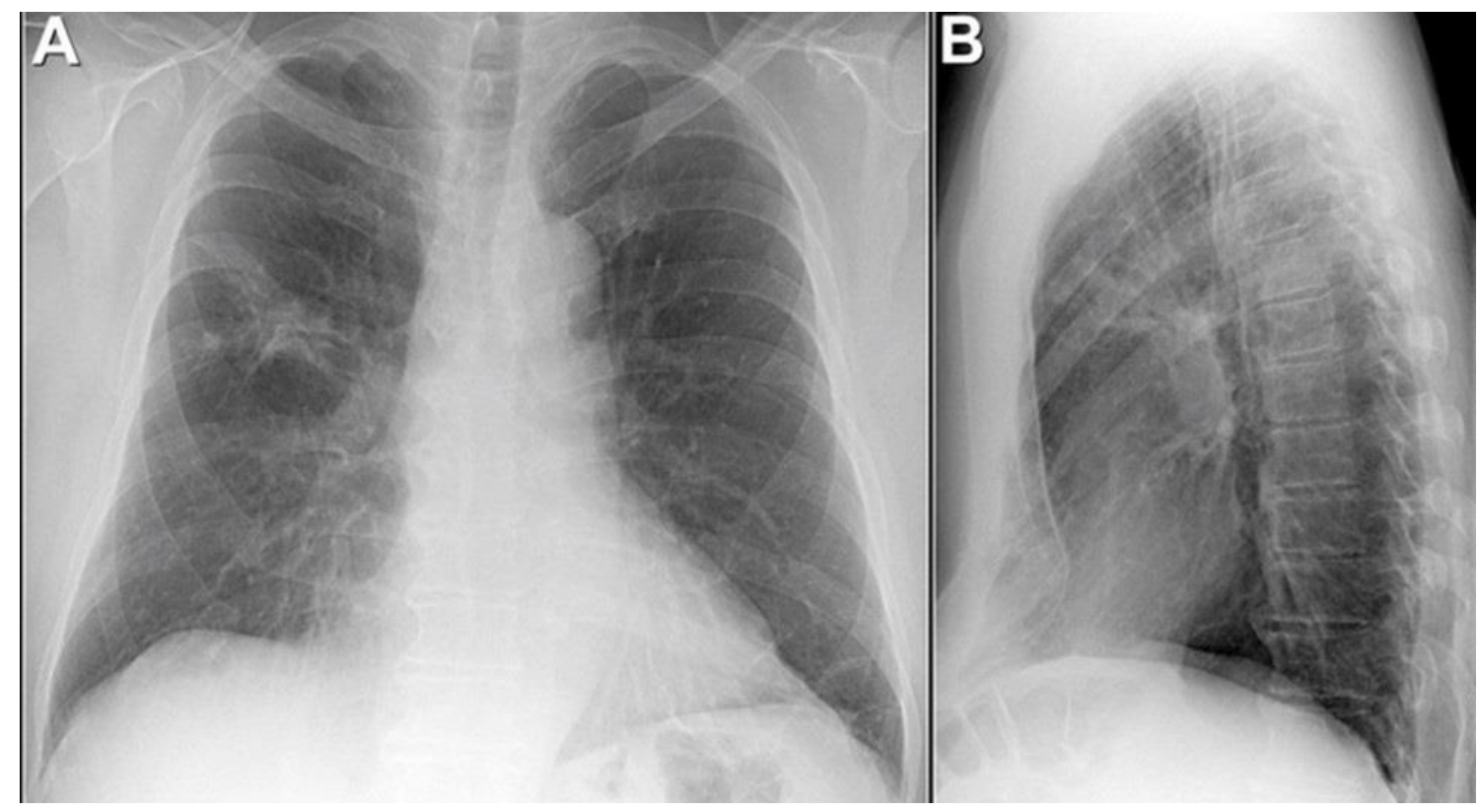

Figure 12. Frontal and lateral chest radiography performed about 1.5 months following Figures 10 and 11.

Which of the following represents the most accurate assessment of the chest radiographic findings?

1. Frontal and lateral chest radiography shows new cavitary lung disease

2. Frontal and lateral chest radiography shows no change in the right upper lobe opacity

3. Frontal and lateral chest radiography shows no interval change from the most recent chest radiograph (Figure 10)

4. Frontal and lateral chest radiography shows progression in the right upper lobe opacity

5. Frontal and lateral chest radiography shows recurrent bilateral lower lobe pneumonia superimposed on the pre-existing right upper lobe opacity 


\section{Correct!}

\section{Frontal and lateral chest radiography shows no interval change from the most recent chest radiograph (Figure 10)}

Frontal and lateral chest radiography (Figure 12) shows no clear change in the opacity within the right upper lobe. No recurrence of the previous bilateral lower lobe pneumonias or nodules is seen, nor is there evidence of cavitation or pleural disease. No other areas of consolidation or new small scattered nodules are evident.).

Pulmonary medicine was consulted and advised repeat unenhanced thoracic CT (Figure 13), maximizing asthma therapy, and monitoring the patient's aortic stenosis.

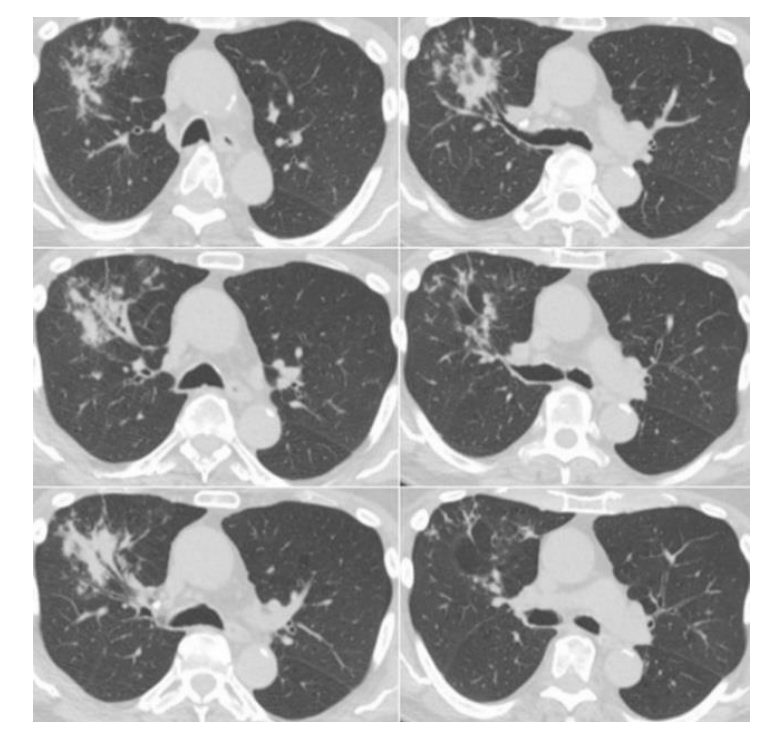

Figure 13. Representative images from axial unenhanced thoracic CT.

Cardiology was consulted and graded the patient's aortic stenosis as "mild-tomoderate," and felt that the aortic stenosis was not responsible, at least entirely, for the patient's intermittent complaints of exertional dyspnea and shortness of breath.

Which of the following represents the most accurate assessment of the thoracic CT findings?

1. Thoracic CT shows development of scattered new small pulmonary miliary nodules

2. Thoracic CT shows marked progression of the anterior segment right upper lobe localized nodular opacities seen on the thoracic CT 2 months earlier (Figure 11)

3. Thoracic CT shows near complete resolution of the right upper lobe nodular opacity

4. Thoracic CT shows recurrence of the bilateral lower lobe pneumonia

5. Thoracic CT shows the right upper lobe opacity appears relatively stable or perhaps minimally improved compared to the thoracic CT 2 months earlier (Figure 11) 


\section{Correct! \\ 5. Thoracic CT shows the right upper lobe opacity appears relatively stable or perhaps minimally improved compared to the thoracic CT 2 months earlier}

(Figure 11)

Repeat thoracic CT (Figure 13) shows that the right upper lobe opacity is relatively stable, perhaps minimally improved, but is neither clearly progressed nor clearly regressed. No new small nodular opacities, pleural disease, or evidence of lymph node enlargement is present.

The patient remained stable over the next two months, after which unenhanced thoracic CT was performed (Figure 14), which was interpreted as worsening of the right upper lobe process.

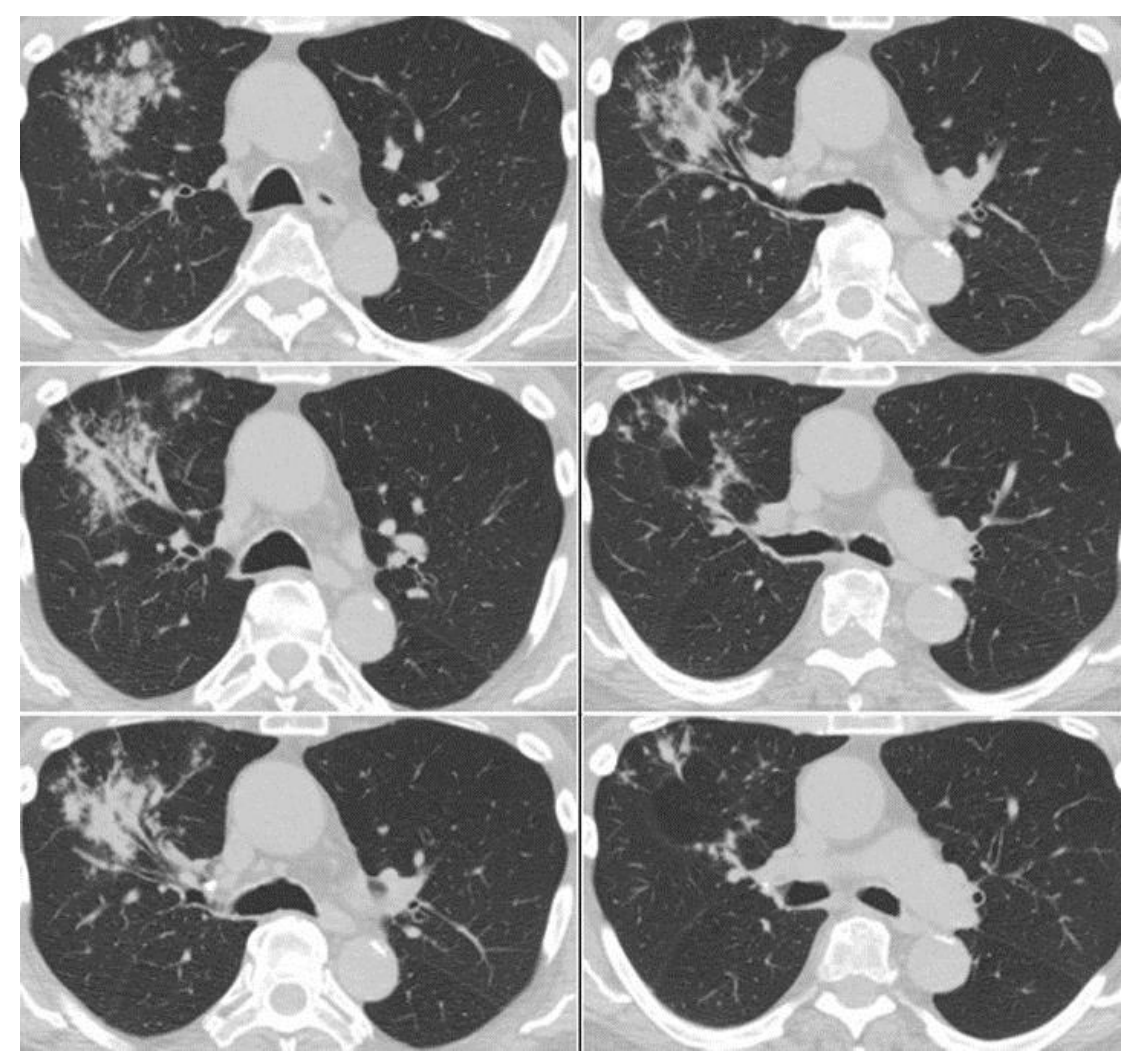

Figure 14. Representative images from axial unenhanced thoracic CT.

At this point, which of the following represents the most appropriate step in this patient's management?

1. Bronchoscopy with transbronchial biopsy

2. Cryobiopsy

3. Open surgical lung biopsy

4. Repeat pulmonary CTA

5. Video-assisted thoracoscopic biopsy 


\section{Correct! \\ 1. Bronchoscopy with transbronchial biopsy}

Repeat pulmonary CTA is unlikely to provide additional data as the right upper lobe opacity has been repeatedly imaged and has shown progression, and the morphology of the process is not suggestive of thromboembolic disease. Both open surgical lung biopsy, and, even more so, video-assisted thoracoscopic biopsy, are reasonable choices, but are needlessly invasive as the diagnosis may potentially be established with bronchoscopy and transbronchial biopsy. Cryobiopsy may eventually play a role for the diagnosis of localized pulmonary opacities, but the limited data for this procedure currently suggests that the primary application of this procedure is in the diagnosis of diffuse fibrotic lung diseases.

The patient underwent bronchoscopy with transbronchial biopsy, which showed a single fragment of alveolar parenchyma without pathologic abnormality. No granulomas, other features of sarcoidosis, viral inclusions, or fungus was seen.

At this point, which of the following represents the most appropriate step in this patient's management?

1. Consult thoracic surgery for diagnostic options for obtaining tissue

2. Cryobiopsy

3. Perform pleuroscopy

4. Repeat bronchoscopy with transbronchial biopsy

5. Treat presumptively for fungal infection and repeat CT in 2 months 


\section{Correct! \\ 1. Consult thoracic surgery for diagnostic options for obtaining tissue}

Repeat bronchoscopy is not unreasonable, but given that this procedure did not yield a diagnosis previously, the likelihood of a repeat procedure providing adequate tissue for a diagnosis, while not zero, is probably low. Therefore, consulting thoracic surgery may be the best course of action, as the patient clearly has a progressive, undiagnosed infiltrative right upper lobe abnormality that could reflect an undiagnosed infection or neoplasm, among other rare proliferative lesions. As noted above, cryobiopsy may eventually play a role for the diagnosis of localized pulmonary opacities, but the limited data for this procedure currently suggests that the primary application of this procedure is in the diagnosis of diffuse fibrotic lung diseases. Continuing to monitor the process will likely be unrewarding, given the imaging evidence of progression, and any presumptive therapy would be merely a guess, without any clear target and therefore the endpoint to therapy would be unclear, and would incur monetary cost and possibly result in complications. Pleuroscopy would not play a role in this patient as he has no discernable pleural disease.

At this point, which of the following represents the most likely diagnosis for this patient?

1. Coccidioidomycosis

2. Organizing pneumonia, likely cryptogenic

3. Primary lung malignancy

4. Sarcoidosis

5. No presumptive diagnosis can be offered 


\section{Correct! \\ 5. No presumptive diagnosis can be offered}

The only data on which a diagnosis could be suggested is the presence of the persistent right upper lobe nodular consolidation. The bronchoscopy with transbronchial biopsy procedure did not provide any specific diagnosis, therefore, this procedure should be regarded as non-contributory. Primary malignancy (bronchogenic, lymphoproliferative) remains a consideration. Coccidioidomycosis is probably unlikely, given that two separate serological assessments for this infection were unrevealing, but the diagnosis is not excluded, and the nodular character of the right upper lobe opacity does raise the possibility of a granulomatous process. For these same reasons, sarcoidosis, while unlikely (particularly since the process appears unilateral), is also not excluded.

Based on the notes in the electronic medical record, the patient was considered to have organizing pneumonia, likely cryptogenic, and corticosteroid therapy with a planned 3-4week taper was instituted, as was azithromycin, with the intention of following the patient clinically and with repeat thoracic CT. Several weeks following bronchoscopy, the patient reported an episode of severe abdominal pain, which woke him from sleep. He was advised to go to the Emergency Room, but instead he presented to his primary care physician several days later with complaints of dizziness and weakness, feeling "terrible," and was found to be tachycardic with orthostatic hypotension on physical examination. His prednisone and azithromycin were discontinued because the patient indicated he always feels "terrible" when taking these medications. The orthostatic hypotension was attributed to the patient's aortic stenosis and some of the medications he was taking (Terazosin taken for hypertension and benign prostatic hypertrophy). The patient continued to periodically see his internal medicine physician, whose notes indicated a working diagnosis of cryptogenic organizing pneumonia. Repeat thoracic CT Images not shown), now 5 months following his most recent CT (Figure 14) was read as progression in the right upper lobe process. The patient continued to see his pulmonary medicine physician, who advised enhanced pulmonary toilet with follow up thoracic CT. The follow up thoracic was performed 7 months following this most recent CT scan (Figure 15). 


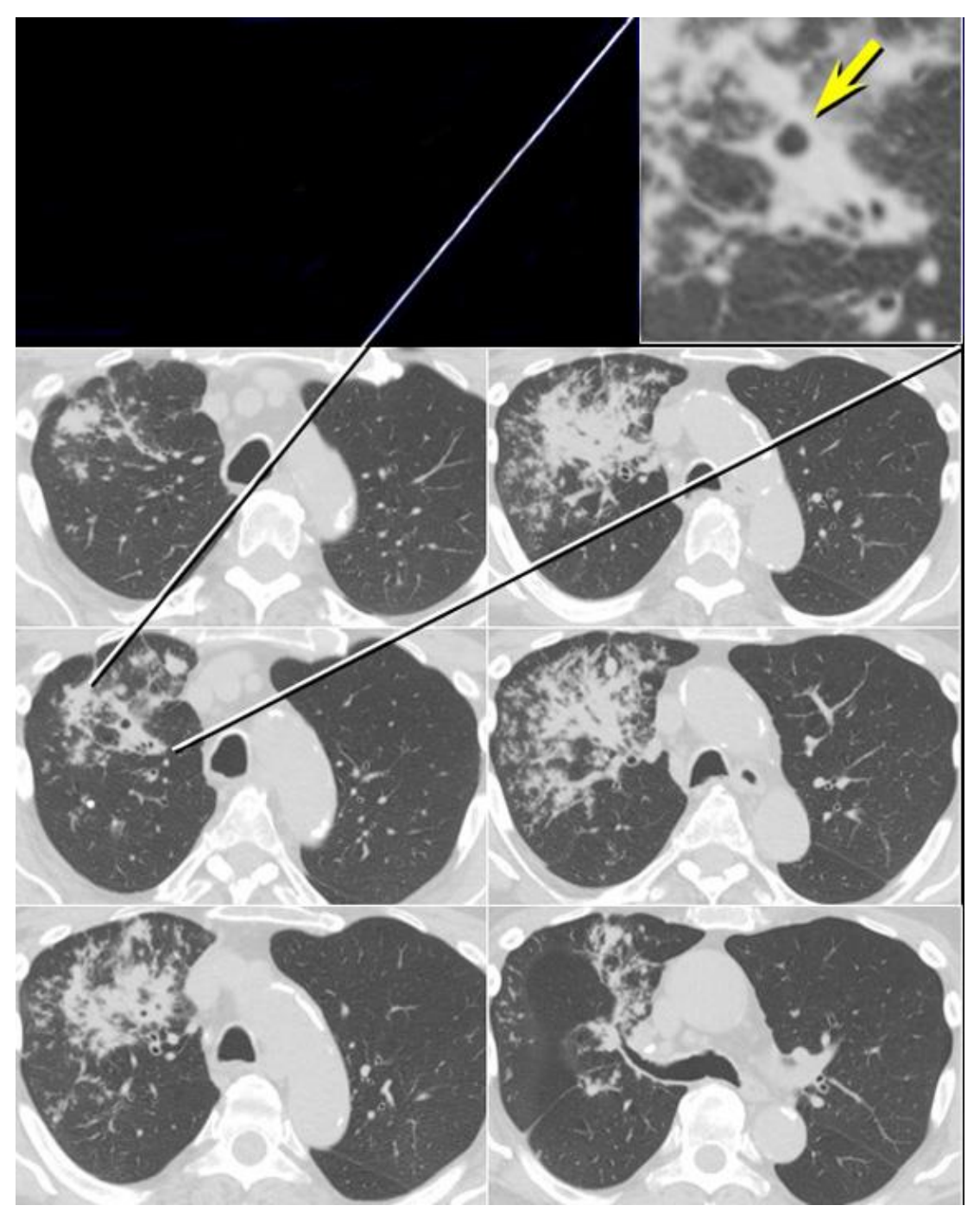

Figure 15: Axial unenhanced thoracic CT now with a small focus of cavitation (yellow arrow in detail image).

Which of the following represents the most accurate assessment of the thoracic CT findings?

1. Thoracic CT shows development of new peribronchial and mediastinal lymph node enlargement

2. Thoracic CT shows development of scattered new small pulmonary miliary nodules

3. Thoracic CT shows progression of the anterior segment right upper lobe localized nodular opacities seen on the thoracic CT 2 months earlier (Figure 11)

4. Thoracic CT shows recurrence of the bilateral lower lobe pneumonias seen previously

5. Thoracic CT shows the right upper lobe opacity appears relatively stable or perhaps minimally improved compared to the thoracic CT 2 months earlier (Figure 11) 


\section{Correct! \\ 3. Thoracic CT shows progression of the anterior segment right upper lobe localized nodular opacities seen on the thoracic CT 2 months earlier (Figure 11)}

The latest repeat thoracic CT (Figure 15) shows that the right upper lobe nodular consolidation has progressed slightly compared to recent CT examinations, and more obviously compared to remote priors, and now even shows a small focus of cavitation. No pleural disease, peribronchial or mediastinal lymph node enlargement, or new disseminated, small nodules are present. However, a number of nodules in the anterior segment of the right upper lobe as well as the right lower lobe, with branching configurations suggesting endobronchial impaction, are evident. Lower lobe opacities with the morphology of the bilateral lower lobe pneumonias seen on what by this point are comparatively remote CT examinations have not recurred.

The patient continued to intermittently present to his primary care physician for similar complaints of shortness of breath, cough productive of white sputum, dizziness, and dyspnea on exertion. During this time frame, the patient was also seen by a number of other consultants for additional complaints, including gastroenterology for dysphagia, otolaryngology for a lesion on his tongue diagnosed as a localized squamoproliferative lesion without dysplasia, general surgery for rib trauma after a mechanical fall, physical medicine for back pain, neurology for altered sensation of taste, and audiology for hearing loss, among others. He continually refused use of prednisone and azithromycin, claiming these medications make him "feel terrible," nor did he follow the recommendations of his pulmonologist for optimized pulmonary toilet and maximization of this asthma therapy. One of his many complains included a $25 \mathrm{lbs}$. weight loss over the previous months, which was attributed to his dysphagia, but CT of the abdomen Figure 16) was obtained to assess for other causes.

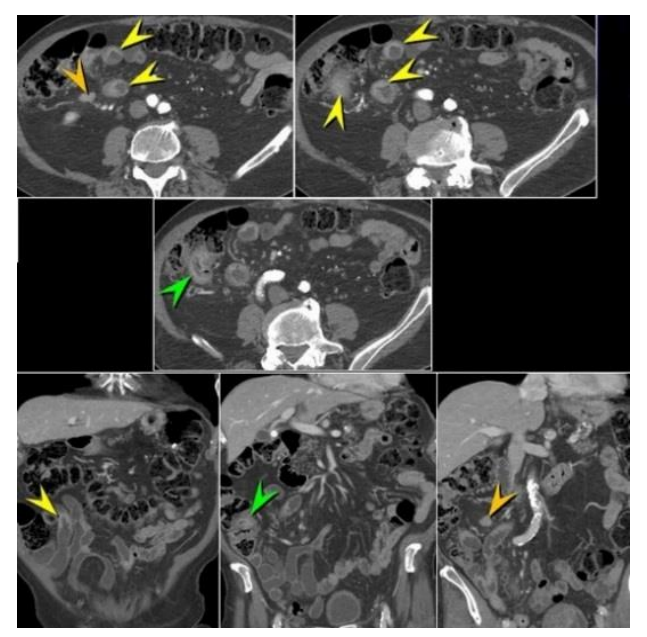

Figure 16: Axial (top and middle row) and coronal (bottom row) images from an enhanced CT of the abdomen show multisegmental, non-contiguous areas of small bowel wall thickening and mucosal hyperenhancement (yellow arrowheads), particularly involving the terminal ileum (green arrowheads). Regional ileocolic lymphadenopathy (orange arrowheads) is present. 
This study showed several short, inflamed segments of small bowel, particularly the terminal ileum.

Which of the following are appropriate differential diagnostic considerations for this patient's condition?

1. Basidiobolomycois

2. Crohn's disease

3. Mycobacterium tuberculosis

4. Ulcerative colitis

5. All of the above 


\section{Correct! \\ 5. All of the above}

All of the entities listed may produce a granulomatous inflammatory process in the small bowel, particularly the ileum. New-onset Crohn's disease or ulcerative colitis would be highly unusual in a patient of this age, but nevertheless possible. Basidiobolomycois is an infection caused by the fungus Basidiobolus ranarum, an emerging invasive fungal infection in desert regions of the U.S. Southwest that may mimic malignancy or inflammatory bowel disease and could present in this manner. Sarcoidosis and histoplasmosis are additional considerations as well.

Which of the following represents the next appropriate management step for the evaluation of this patient?

1. Assessment for stool pathogens

2. Inflammatory bowel disease markers

3. Repeat colonoscopy using sterile specimen collection methods

4. Surgical exploration of the abdomen

5. All of the above 


\section{Correct! \\ 5. All of the above}

Assessment for inflammatory bowel disease markers [such as neutrophil specific antigen, Saccharomyces cerevisiae $\lg A$ and $\lg G$ antibody, C-reactive protein, and the erythrocyte sedimentation rate), repeat colonoscopy to obtain samples for infection, and assessment of the stool for various fungal and mycobacterial pathogens are appropriate measures to pursue.

All the inflammatory bowel disease serologies were positive. Repeat colonoscopy using techniques to collect samples for infection did not reveal fungus or mycobacteria, nor was there evidence of viral infection, such as cytomegalovirus or herpes simplex, although the repeat biopsy again showed non-necrotizing granulomatous inflammation similar to what was seen at the first colonoscopy procedure. The cecum could not be intubated due to the presence of a stricture. No Splendore-Hoeppli phenomenon (asteroid bodies) - the in vivo formation of intensely eosinophilic material around microorganisms (fungi, bacteria and parasites) or biologically inert substances- was seen. A QuantiFERON test was negative. Characteristic features of basidiobolomycosis, including an eosinophilic-rich infiltrate in the biopsied material and peripheral eosinophilia, were lacking. During this period when the next steps in management were being contemplated, stool culture was reported positive for mycobacteria, speciated as Mycobacterium tuberculosis, subsequently shown to be pan-sensitive. Four-drug therapy employing rifampin, isoniazid, ethambutol, and ezetimibe with B6 supplementation was begun. Subsequent bronchoscopy with bronchial lavage also showed acid-fast bacilli with growth in culture and treatment was continued with the patient in respiratory isolation.

Diagnosis: Mycobacterium tuberculosis infection of the lungs with dissemination to the terminal ileum

\section{References}

1. Cardinale L, Parlatano D, Boccuzzi F, Onoscuri M, Volpicelli G, Veltri A. The imaging spectrum of pulmonary tuberculosis. Acta Radiol, 2015; 56(5):557-64. [CrossRef] [PubMed]

2. Prapruttam D, Hedgire SS, Mani SE, et al. Tuberculosis the great mimicker. Semin Ultrasound CT MR. 2014;35:195-214. [CrossRef] [PubMed]

3. Vikram HR, Smilack JD, Leighton JA, Crowell MD, De Petris G. Emergence of gastrointestinal basidiobolomycosis in the United States with a review of worldwide cases. Clin Infect Dis. 2012;54(12):1685-91. [CrossRef] [PubMed\}

4. Lee JH, Han DH, Song JW, et al. Diagnostic and therapeutic problems of pulmonary tuberculosis in elderly patients. J Korean Med Sci. 2005; 20:784-9. [CrossRef] [PubMed]

5. Jung $Y$, Hwangbo $Y$, Yoon SM, et al. Predictive factors for differentiating between Crohn's disease and intestinal tuberculosis in Koreans. Am J Gastroenterol. 2016; 111(8):1156-64. [CrossRef] [PubMed] 
6. Leung AN. Pulmonary tuberculosis: the essentials. Radiology. 1999;210(2):307-22. [CrossRef] [PubMed]

7. Rozenshtein A, Hao F, Starc MT, Pearson GD. Radiographic appearance of pulmonary tuberculosis: dogma disproved. AJR Am J Roentgenol. 2015;204(5):9748. [CrossRef] [PubMed] 